\title{
Wilfried LIGNIER, Prendre. Naissance d'une pratique sociale élémentaire
}

Sophie Richardot

\section{(2) OpenEdition}

1 Journals

Édition électronique

URL : http://journals.openedition.org/ress/6167

DOI : $10.4000 /$ ress.6167

ISBN : $1663-4446$

ISSN : 1663-4446

Éditeur

Librairie Droz

Édition imprimée

Date de publication : 6 juillet 2020

Pagination : $271-275$

ISSN : 0048-8046

Référence électronique

Sophie Richardot, «Wilfried LIGNIER, Prendre. Naissance d'une pratique sociale élémentaire », Revue européenne des sciences sociales [En ligne], 58-1 | 2020, mis en ligne le 06 juillet 2020, consulté le 01 décembre 2020. URL : http://journals.openedition.org/ress/6167 ; DOI : https://doi.org/10.4000/ress. 6167

Ce document a été généré automatiquement le 1 décembre 2020.

(c) Librairie Droz 


\title{
Wilfried LIGNIER, Prendre. Naissance d'une pratique sociale élémentaire
}

\author{
Sophie Richardot
}

\section{RÉFÉRENCE}

Wilfried LIGNIER, 2019, Prendre. Naissance d'une pratique sociale élémentaire, Paris, Éditions du Seuil, 328 p.

1 Avec Prendre. Naissance d'une pratique sociale élémentaire, Wilfried Lignier investit une question jusque-là délaissée par la sociologie: la manière dont très jeunes enfants s'approprient les objets de leur environnement. L'échelle d'analyse est focalisée sur les gestes, un niveau de réalité habituellement réservé au champ des sciences cliniques ou expérimentales. Pourtant, selon l'auteur, il est pleinement légitime, pour les sciences sociales, de s'intéresser à cette question qui interroge tout aussi bien l'inégale distribution des biens accessibles dans la population que le rôle des institutions dans la formation des préférences enfantines ou la manière dont les goûts et les dégoûts créent de l'affiliation ou de la ségrégation sociale. L'originalité de l'ouvrage - et de l'enquête qu'il présente - réside ainsi dans son projet même : explorer à nouveaux frais ce qui, à première vue, peut apparaître comme une "petite question" pour en révéler les enjeux sociaux fondamentaux.

2 La thèse est la suivante : « ce que nous tenons sans aucun doute de la nature, ce qui est irrémédiablement en nous depuis la naissance, c'est un désir générique d'appropriation de ce qui nous entoure. Mais l'intensité de notre tendance à prendre lors de nos premières années d'existence, ce que nous cherchons à prendre effectivement, ainsi que la manière dont nous avons tendance à procéder, au moment même de l'acte, tout cela est forcément particulier, c'est-à-dire particularisé par des conditions locales singulières: une époque, un milieu, un moment, une interaction » (p.17). Dès lors, l'acte de «prendre » doit être analysé comme un processus social plutôt que naturel. Il convient de l'étudier dans son contexte ordinaire, plutôt qu'en laboratoire. C'est ce que 
propose ici l'auteur en nous livrant les résultats d'une recherche ethnographique minutieuse menée dans une crèche parisienne. Sur le plan théorique, Wilfried Lignier mobilise la théorie de Pierre Bourdieu pour rappeler que les objectifs de l'action ne sont pas les seules à importer dans la vie sociale et que la notion de pratique renvoie également à la manière de faire, au style de l'action. Il s'agit dès lors, pour lui, de s'intéresser non seulement aux visées de l'appropriation enfantine mais également aux manières différenciées de prendre et aux types de preneurs pour comprendre, au fond, la sociogenèse de l'habitus et du sens pratique. L'ouvrage, dense, se compose de cinq chapitres bien articulés et se caractérise par sa grande cohérence théorique et empirique.

3 Le premier chapitre - «De l'expérimentalisme à l'ethnographique » - est consacré à une critique approfondie des études expérimentales en matière d'approche scientifique du jeune âge. Pour Wilfried Lignier, il faut se distancier, d'une part, d'une approche comportementale de la préhension qui envisage les prises comme un geste universel, principalement déterminé par les conditions physiques immédiates de sa réalisation et, d'autre part, d'une approche psychologique du sens de la propriété qui prend pour point de départ la subjectivité individuelle et ses nécessité propres (agir au mieux, se rassurer, etc). L'une et l'autre réduisent l'intervention du social, soit parce qu'elles décontextualisent les pratiques enfantines, soit parce qu'elles font intervenir l'histoire et la culture beaucoup trop tard dans le processus de développement et, dans tous les cas, parce qu'elles se préoccupent trop peu de la différenciation sociale entre enfants. On saluera ici ce qui est très rarement fait ailleurs : un véritable travail d'analyse et de discussion des travaux de psychologie expérimentale conduisant à des critiques argumentées, et fondées, des recherches conduites dans ce domaine. On peut néanmoins regretter qu'un état de l'art ne soit pas ensuite proposé à partir des études menées en psychologie culturelle, un champ de recherches qui intègre pleinement l'auteur le reconnaît lui-même - la dimension sociale dans ses analyses.

Wilfried Lignier insiste ensuite sur la nécessité de sortir du laboratoire pour observer, sur le vif, les prises enfantines ordinaires et présente son enquête ethnographique. Il s'agit, tout en réinscrivant l'analyse conduite sur le terrain dans des dynamiques sociales plus générales (historiques, institutionnelles, de morphologie sociale, etc.), de mener en crèche de très fines observations : quelles choses les enfants prennent-ils ? Comment y accèdent-ils? Quelles expressions accompagnent les prises? Quelle place joue la violence dans l'accès aux choses? On peut s'étonner que l'auteur n'ait pas eu recours à l'usage de la vidéo car celle-ci, en dépit de ses contraintes (surmontables), aurait été particulièrement utile pour la possibilité qu'elle offre d'appréhender une scène dans toute sa complexité (ce qui est d'autant plus précieux à propos de pratiques enfantines encore relativement indéterminées) et, surtout, de l'appréhender autrement que sur le vif (ce qui permet à l'observateur de réviser éventuellement son premier regard qui tend souvent à " remettre de l'ordre » là où il n'y en a pas forcément). Il n'en demeure pas moins que les objectifs de l'observation sont clairement définis et que l'ensemble du processus de "prise » est ici scrupuleusement analysé. L'enquête a été conduite dans la section des "grands » d'une crèche municipale parisienne (28 enfants de 2 à 3 ans), relativement mixte socialement (8 enfants sont d'origine populaire, dont 6 issus de l'immigration ; 6 issus des classes moyennes; 14 des classes supérieures), et s'est étalée sur toute l'année. Elle a été complétée par des entretiens auprès de la plupart des parents, généralement au domicile de ceux-ci, et auprès des professionnelles de la crèche. Le chercheur a également eu accès aux notes prises par 
les professionnelles ainsi qu'aux dossiers individuels des enfants. Les matériaux recueillis sont donc très riches. Sans doute l'échantillon permet-il d'adopter une perspective variationniste sur le jeune âge mais on peut néanmoins penser que celle-ci aurait été renforcée si l'enquête ne s'était pas concentrée sur une seule crèche et avait pu être conduite dans une autre structure.

5 Le deuxième chapitre - «En crèche : dans le domaine du prenable » - fait entrer le lecteur sur le terrain d'enquête. Il s'agit de s'intéresser aux prises enfantines en termes de disponibilité des choses (à prendre) et de dispositions des enfants (qui prennent). Comment se distribuent les objets et les espaces autour des enfants? Qui contrôle cette distribution? L'auteur montre combien la disponibilité des choses est instituée a priori (en laissant, par exemple, certaines choses à portée de main tandis que d'autres sont placées loin des enfants, en délimitant des moments d'appropriation, en encourageant des pratiques légitimes, etc.). Tout concourt, en particulier, à ce que les appropriations matérielles soient les plus pacifiques possibles. Pour tenter d'analyser les dispositions des preneurs, l'auteur s'attache à mettre en relation des différences relatives au cadre de socialisation familial et des différences de comportements en crèche. L'enquête conduite au domicile familial lui permet de formuler une hypothèse intéressante : les enfants des classes moyennes et supérieures sont plus détendus, plus confiants dans leur pratique d'appropriation (car ils évoluent dans des univers où les objets sont nombreux et accessibles) tandis que ceux des classes populaires sont davantage pris par l'urgence et l'inquiétude (en raison de la rareté des objets et de d'espace auxquels ils accèdent). Mais l'impatience observée chez les enfants d'origine populaire vis-à-vis des choses (que l'on est sur le point de leur distribuer, par exemple) ne peut-elle pas s'expliquer également par une socialisation différenciée au temps comme le montrent certaines recherches récentes (Gaële Henri-Panabière et al., 2019, «La montre et le martinet : structuration temporelle de la vie familiale et inégalités scolaires ", Actes de la recherche en sciences sociales, 226-227, 1, p. 16-30)?

6 Le troisième chapitre - «Ce que les enfants préfèrent prendre » - s'intéresse à ce qui conduit de jeunes enfants à préférer certains objets plutôt que d'autres dans leurs pratiques d'appropriation. Wilfried Lignier constate que les enfants sont attirés, à la crèche, par des objets qu'ils ont appris à manipuler à la maison, mais, également, que leurs préférences se construisent au cours de leurs interactions quotidiennes. "Être très jeune, c'est avoir des dispositions personnelles encore suffisamment peu arrêtées pour laisser la pratique assez ouverte » (p. 154). C'est ainsi que les enfants se saisissent d'objets sur lesquels l'adulte attire leur attention, ici et maintenant, ou s'orientent, de manière plus ou moins accidentelle, vers ce qui est accessible. Les observations suggèrent ainsi que les enfants ne prennent pas tant ce qu'ils préférent a priori mais ont plutôt tendance à rendre préférable a posteriori ce qu'ils ont d'abord pris un peu par hasard.

7 Le quatrième chapitre - «Comment prendre par soi-même quand on est tout petit ? traite ensuite des façons de prendre et du recours, par les enfants, à une certaine violence, physique ou symbolique, pour accéder à ce qu'ils veulent. S'agissant de la violence physique, l'enquête montre que son usage est à la fois largement exploré par les enfants mais aussi limité, car il est immédiatement sanctionné et disqualifié par les professionnelles de la crèche. S'agissant de la violence symbolique, l'auteur observe un phénomène particulièrement intéressant : les enfants ont tendance à reprendre à leur compte, en les détournant à leur profit, les formules, souvent injonctives, qui leur ont 
été adressées par les adultes. Ils vont déclarer, par exemple, «je partage » pour justifier qu'ils s'emparent de ce que les autres détiennent. Wilfried Lignier et Julie Pagis (L'Enfance de l'ordre, 2017, Paris, Éditions du Seuil) ont proposé de nommer ce phénomène recyclage car "il s'agissait, en choisissant ce terme (plutôt que ceux de répétition ou de transposition, qui auraient été également envisageables) d'insister sur l'innovation relative des enfants socialisés, dans leur reprise symbolique» (p. 236). L'auteur souligne que violence physique et violence symbolique ne sont pas exclusives mais il ressort néanmoins de l'enquête que certains enfants - souvent d'origine populaire - ont une tendance marquée à taper, pousser, frapper pour prendre tandis que, d'autres - de classes favorisées - sont plus souvent engagés dans des modalités verbales ou symboliques d'imposition.

8 Le cinquième et dernier chapitre - "L'appropriation des autres, premiers essais » montre que la prise des choses n'est pas étrangère à l'appropriation des personnes, qui peuvent être vues comme de "super-choses" par les enfants. Plus précisément, l'auteur se demande comment l'appropriation des autres - l'établissement délibéré d'alliances personnelles - repose sur la capacité à donner des choses que l'on a. Certains enfants sont-ils davantage capables que d'autres de s'allier aux autres? Peuton repérer des liaisons préférentielles ? Les résultats, obtenus à partir d'une analyse de réseau, font apparaître une très forte sexuation des alliances observées entre enfants et une tendance des filles à former des groupes plus cohésifs et plus constitués que les garçons. Wilfried Lignier formule, à ce propos, une hypothèse prometteuse : «les espaces féminins d'élection (ceux qui font écho à leurs dispositions déjà distinctives) [l'espace " dînette", " coiffure ", etc.] sont bien faits pour favoriser le croisement répété de plusieurs filles s'activant toutes ensemble dans un endroit fixe et relativement clos; à l'inverse, les espaces masculin d'élection, plus labiles, plus flous, plus mouvants, limite a priori la proximité physique véritablement collective, au profit de rapprochement entre garçons se réalisant deux à deux, autour d'un objet plutôt que d'un lieu » (p. 279). Le chapitre montre très finement comment les enfants, constatant l'inefficacité du recours à la force pour s'approprier les personnes, en viennent à adopter des conduites alternatives, mieux à même de retenir l'attention, de susciter la bonne volonté, l'assistance ou la coopération d'autrui. Mais peut-on encore parler d'« appropriation » quand l'enfant prend en compte l'envie de l'autre ou quand les interactions deviennent, avec l'âge, plus coopératives?

9 L'auteur rappelle, en conclusion, que «le geste qui nous porte vers les choses est un geste social» (p. 305) ainsi que le produit d'une histoire sociale «faite corps». L'observation ethnographique révèle, en particulier, l'incorporation précoce des dispositions sexuées et les alliances, déjà ségrégatives, qui en découlent. Wilfried Lignier insiste également sur le rôle crucial de l'adulte en particulier dans l'attribution d'une valeur aux choses. Il souligne aussi l'importance du langage dans l'appropriation des objets par les enfants et, les inégalités qui en résultent, tant les différences de compétences langagières apparaissent tôt. Il s'interroge sur les rapports entre possession et violence ainsi que sur la durée de vie des pratiques qu'il a observées. Sous quelle forme subsistent-elles avec l'avancée en âge ? Enfin, à partir du constat selon lequel «les enfants se comportent comme si importait constamment pour eux la distinction dominant/dominés » (p.316), il conclut que «les logiques de distinction n'interviennent pas de façon secondaire dans la sociogenèse de la pratique; elles en sont constitutives, dès le début » (p. 316). 
10 On ne peut que recommander la lecture de Prendre dont l'apport dans le champ de la sociologie de l'enfance est indéniable, même si on peut se demander pour quelles raisons Wilfried Lignier n'a pas davantage mobilisé les apports de la psychologie culturelle du développement (voir, par exemple, Richard. A. Shweder et al., "The Cultural Psychology of Development: One Mind, Many Mentalities », in William Damon \& Richard M. Lerner (eds), 2016, Handbook of Child Psychology, Hoboken (NJ), Wiley, vol. 1, p. 716-792), dont la posture épistémologique et méthodologique est proche de celle qu'il défend. Un tel dialogue aurait, certes, impliqué de renoncer à une certaine stratégie de distinction disciplinaire, mais il aurait pu être particulièrement fécond et permis de conforter l'assise théorique de la recherche. Espérons, enfin, que ce livre sera aussi lu par les professionnels de la petite enfance et par les tenants d'une approche purement expérimentale du premier âge, dont Wilfried Lignier souligne à juste titre, les impensés et les limites.

\section{AUTEURS}

SOPHIE RICHARDOT

CURAPP-ESS, CNRS, Université de Picardie-Jules Verne 